\title{
Modeling of rhythmic behavior in neutral lipid production due to continuous supply
} of limited nitrogen: Mutual growth and lipid accumulation in microalgae \author{
Rahul Tevatia ${ }^{1,2, *}$, James Allen $^{3}$, Paul Blum², Yaşar Demirel ${ }^{1}$, Paul Black ${ }^{3}$ \\ 1- Department of Chemical and Biomolecular Engineering, University of Nebraska-Lincoln, \\ Lincoln NE 68588, USA \\ 2- School of Biological Sciences, University of Nebraska-Lincoln, Lincoln NE 68588, USA \\ 3- Department of Biochemistry, University of Nebraska-Lincoln, Lincoln NE 68588, USA \\ * - rahultevatia_83@yahoo.co.in
}

\begin{abstract}
The relative effects of three precise nitrogen limitation regimes on green microalgae were assessed using the Trebouxiophycean alga Coccomyxa subellipsoidea grown in a chemostatic bioreactor system. The data provides further evidence that growth and triglyceride (TAG) accumulation are concurrent and independently proportional to the degree of nitrogen limitation in algae. Additionally, TAG accumulation was observed to proceed via oscillations with respect to time and percent dry weight quantity. The predator-prey model was applied to fit the experimental data and to obtain the physiological significance of these oscillations. The results determine the conditions of maximum neutral lipid productivity with respect to nitrate stress and highlight an area of potential future research.
\end{abstract}

Keywords: Continuous nitrogen limitation; Microalgal growth; Neutral lipid accumulation; Prey - predator model; Maximum productivities. 


\section{Introduction}

Microalgae could be a potential source of sustainable biofuel (Chisti and Yan, 2011). They display a clear advantage of high biomass and lipid productivity compared to other biofuel crops, though the lipids yields are strictly strain dependent (Brennan and Owende, 2010). Further, microalgae can be grown photoautotrophically, mixotrophically, heterotrophically and/or photoheterotrophically (Brennan and Owende, 2010; Gouveia and Oliveira, 2009). It is well established that nitrogen stress results in neutral lipid accumulation, but at the cost of decreased microalgae growth and reduced biomass (Breuer et al., 2012; Tevatia, et al., 2012; Yeh and Chang, 2011). Most of these studies of nitrogen stress were done in closed systems like including photobiorectors and raceways ponds (Chisti and Yan, 2011; Brennan and Owende, 2010). The most effective technique to produce maximum yields was found to be cultivating microalgae for sequential growth and starvation (Prathima et al., 2012; Das et al., 2011). The method is effective, but time consuming and costly. Therefore, producing high biomass with high triacylglycerol (TAG) demands an efficient and effective system.

Algal continuous cultures have commonly resulted in negligible neutral lipid yields leading to their widespread dismissal as viable platforms for biofuel production (Richardson et al., 1969; Hoffmann et al., 2010; Lacour et al., 2012). However, two recent studies have demonstrated the possibility of using this culturing method to tune algal growth and lipid accumulation through precision nutrient limitation (Klok et al., 2013; Adams et al., 2013). Klok et al. (2013) observed simultaneous growth and lipid accumulation when Neochloris oleoabundans was grown in a flat panel photobioreactor under nitrogen limited turbidostatic conditions and suggested that TAG synthesis serves 
as energy sink for the maintenance of photosynthetic viability. Similarly, Adams et al. (2013) performed the batch and semi-continuous studies on six different strains of microalgae with precise nitrogen amounts, and observed both biomass and lipid to be increasing with time. The current study elaborates on these efforts by analyzing the relationship between growth, TAG accumulation, and precision nitrogen limitation using chemostatic continuous culture with the ultimate goal of tailoring the output of biomass and its TAG concentration.

The present study exhibits (i) concurrent growth and lipid accumulation, and (ii) the oscillating patterns in lipid production in Coccomyxa subellipsoidea, a recently sequenced Trebouxiophycean micro-algae currently used as a model system for biofuelsrelated lipid biochemistry, when the cells were grown in continuous photobioreactor with precise limited nitrate addition. In order to avoid any chaos in the model, the system was forced with continuous supply of light (escaping circadian oscillations due to light-dark cycles). Oscillatory behavior has been long studied in the living systems, and found to be a result of various cellular regulations (Goldbeter, 2002). Therefore, the complexity of the biological process involved to generate oscillations was in need of an explanation in order to understand the nature of these rhythms. Hence, model in terms of prey-predator model was used to understand the nonlinear dynamics of these oscillations.

\section{Materials and Methods}

\subsection{Microalgae strain and growth conditions}

Coccomyxa subellipsoidea strain C169 strain obtained from Dr. James Van Etten (University of Nebraska-Lincoln) was used for the present study and grown under 
photoautotrophic conditions in Bold's Basal Medium (BBM) at pH 6.70 (Bold, 1949).

The pre-culture for the continuous experiments were prepared separately in a $3 \mathrm{~L}$

photobioreactor to achieve maximum cell density under controlled condition of

continuous illumination ( $200 \mu \mathrm{mol}$ photons $\left.\mathrm{m}^{-2} \mathrm{~s}^{-1}\right)$, agitation $(500 \mathrm{rpm})$, and $\mathrm{pH}(6.70)$ at $25^{\circ} \mathrm{C}$.

\subsection{Continuous photobioreactor setup}

Applikon double-jacketed 3.0 L bioreactor (Applikon Biotechnology Inc., USA) was

used (Fig. 1). Continuous illumination of $200 \mu$ mols photons $\mathrm{m}^{-2} \mathrm{~s}^{-1}$ was provided by 85 W SHO bulb (American Aquarium Products). The uniformity of initial light intensity was confirmed by measuring light in the empty photo-bioreactor (with distilled water in the outer jacket) using a quantum meter (Li-Cor, Lincoln, NE, USA). One impeller with two Rushton turbine blades, and three baffles were used for proper mixing. Temperature was maintained by exchanging the heat to media by running water in the outer jacket attached to a thermocirculator. Autoclavable $\mathrm{pH}$ electrode (Applikon Biotechnology Inc., USA), dissolved oxygen probe (Applikon Biotechnology Inc., USA), and biomass monitor (Optek-Danulat Inc., USA) were used to monitor $\mathrm{pH}$, dissolved oxygen (DO), and biomass production, respectively in real-time. All real-time data of photobioreactor were stored in connected to the computer (Dell, USA) using bioexpert software of Applikon. The working volume of $2.0 \mathrm{~L}$ was used for all continuous studies. The reactor temperature and stirrer speed were maintained at $25^{\circ} \mathrm{C}$ and $500 \mathrm{rpm}$, respectively. The condenser temperature was maintained at $4{ }^{\circ} \mathrm{C}$ to cool down the water vapors, in order to prevent any evaporation loss during the runs. The $\mathrm{pH}$ of BBM was maintained at 6.7 
using $0.1 \mathrm{M} \mathrm{HCl}$ and $0.1 \mathrm{M} \mathrm{KOH}$. The medium was aerated with compressed air (containing $390 \mathrm{ppm} \mathrm{CO} 2$ as carbon-source) at a rate of $1 \mathrm{vvm}$ (one volume of air per liter culture in one minute) through $0.2 \mathrm{~mm}$ polytetrafluorothylene (PTFE) filter. Finally, the medium was inoculated with pre-culture prepared in Section 2.1.

The continuous photobioreactor was maintained for 40 days ( 10 days in each dilution for the total of four dilution rates). As shown in Fig. 1, the input tubing (Masterflex, Cole-Parmer, USA) was connected to the reservoir containing the BBM media with 833.0 $\mu \mathrm{M}$ nitrate, and the output tubing to the collecting vessel $(500 \mathrm{ml})$ in a refrigerator (a small hole was created on the roof of refrigerator for inserting tubing and then closed with a seal). Both input and output tubing were passed thorough a common peristaltic pump (in order to maintain equal input and output flow rates). The speed of peristaltic pump was controlled by bioexpert software after connecting the pump to the ezcontroller (Applikon Biotechnology Inc., USA). The nitrate availability to the photobioreactor was determined by the inflow of external media containing $833.0 \mu \mathrm{M}$ nitrate. The flow rates were adjusted so that the targeted nitrate addition rate was achieved. The special attention was given for the attainment of sterile conditions, which was regularly checked by microscopy. Started from the dilution rate corresponding to highest limited nitrate addition rate $\left(194.0 \mu \mathrm{g} \mathrm{L} \mathrm{L}^{-1} \mathrm{~h}^{-1}\right)$ were dropped to second dilution (129.3 $\left.\mu \mathrm{g} \mathrm{L}^{-1} \mathrm{~h}^{-1}\right)$ and finally to the lowest $\left(64.7 \mu \mathrm{g} \mathrm{L}^{-1} \mathrm{~h}^{-1}\right)$ limited nitrate addition rate, the samples were collected after every $24 \mathrm{~h}$ for OD550, dry weight, and neutral lipid analysis. The final dilution (fourth dilution, $388.0 \mu \mathrm{g} \mathrm{L} \mathrm{L}^{-1} \mathrm{~h}^{-1}$ ) was set to mimic the replete condition, to monitor the effect for the change of limited to replete state on the cells. The used dilution rates and corresponding nitrate addition rates are listed in Table 1. 
Bioreactor runs were replicated twice.

\subsection{Biomass analysis}

Samples taken from the bioreactor were centrifuged at $2960 \times \mathrm{g}$ for $15 \mathrm{~min}$. Pellet was then washed thrice with distilled water and lyophilized in freeze dry system (Labconco Corp., USA). The lyophilized powder was used for dry weight analysis.

\subsection{Confocal microscopy}

The lipophilic fluorophore Nile Red was used to stain the cells for assessing the effect of nitrogen limitation on neutral lipid accumulation as previously described (Chen et al., 2009). The fluorscence, and transmitted light images were acquired with a laser scanning confocal microscope (Olympus Fluoview 500), using 100x oil immersion lens, and analyzed with the Fluoview (v4.3) software.

\subsection{Total lipid extraction and triglyceride quantification by LC-MS/MS}

Total lipids were extracted using the method of Bligh and Dyer (Bligh and Dyer, 1959) with some modifications. Samples were first disrupted using a bead mill (Qiagen Tissue-Lyser LT, Qiagen, Valencia, CA), methanol:chloroform (2:1) containing 0.01\% butylated hydroxytoluene (BHA), and approximately $250 \mathrm{mg}$ of $0.7 \mathrm{~mm}$ zirconia beads (Biospec Products, Bartlesville OK) twice for 5 min. A triglyceride internal standard (TG $(17: 1 / 17: 1 / 17: 1))$ was added prior to disruption. The samples were vortexed at low speed for $1 \mathrm{hr}$ in $3 \mathrm{~mL}$ of extraction solvent followed by the addition of $1 \mathrm{~mL}$ each of saturated aqueous sodium chloride solution and chloroform to induce phase separation. The vials 
were centrifuged at $2500 \mathrm{x}$ g for $5 \mathrm{~min}$ and the solvent partitions transferred to fresh glass vials. These were dried with a nitrogen gas evaporator (Organomation, Berlin, MA) heated to $45^{\circ} \mathrm{C}$ and suspended in $1 \mathrm{~mL}$ of methanol/chloroform $(1: 1)$ for storage in sealed glass vials under argon gas. Samples were stored at $-80{ }^{\circ} \mathrm{C}$ until analyzed using an AB SCIEX Q-Trap 4000 LC-MS/MS with a Shimadzu UFLC-XR and the quantification method of Allen et al., 2014. Briefly, $100 \mu \mathrm{L}$ aliquots of the lipid extracts were reacted by vortexing at low speed with hydrogen gas in the presence of $1 \mathrm{mg}$ of platinum IV oxide and $0.5 \mathrm{~mL}$ of chloroform for $30 \mathrm{~min}$. Liquid chromatographic separations were obtained using an SB-C18 $3 \mu \mathrm{m} 2.1$ x 100 mm column (Agilent Technologies, Santa Clara, CA) and a two solvent elution of methanol with formate $(1.6 \mathrm{mM} ; 0.01 \%)$ and formic acid (0.7 mM; 0.004\%) added (solvent A) and chloroform (solvent B). Saturated triglycerides were quantified by comparing peak areas from multiple reaction monitoring of the $\left[\mathrm{M}+\mathrm{NH}_{4}\right]^{+}$adducts and $[\mathrm{M}+\mathrm{H}]^{+}$diacylglyceride fragments of sample analytes with the internal standard.

\section{Results and Discussion}

\subsection{Mutual growth and neutral lipid accumulation}

\subsubsection{Effect of continuous supply of limited nitrates}

To determine the effect of the precise amount of continuous limited nitrate addition on the growth and lipid accumulation, C. subellipsoidea was grown in four different dilution rates representing four different nitrate addition rates to the photobioreactor. A schematic of the continuous photobioreactor setup is presented in Figure 1. Each dilution 
was studied for 10 days, and then adjusted to next dilution rate. Samples were collected, centrifuged and then kept in $-80{ }^{\circ} \mathrm{C}$ for further analysis.

Figure 2 summarizes the complete continuous photobioreactor study performed for a period of 40 days. Photobioreactors experiments were duplicated and all individual analysis was done in triplicates. Three subsequent dilutions, which resulted in limited nitrate condition, were adjusted after every $10^{\text {th }}$ day, and the final fourth dilution rate was maintained in excess nitrate to reflect the replete condition. These effects are presented and discussed in following sections:

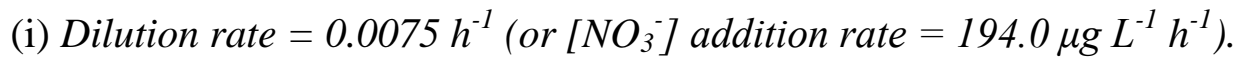

At this nitrate addition rate, the biomass concentration was detected to be constant (a turbidostat state) $\sim 350 \mathrm{mg} \mathrm{L}^{-1}$ and neutral lipid was observed to be oscillating between $2.05 \pm 0.23$ and $2.79 \pm 0.20 \%$ DW (dry weight).

(ii) Dilution rate $=0.0050 h^{-1}\left(\right.$ or $\left[\mathrm{NO}_{3}^{-}\right]$addition rate $\left.=129.3 \mu \mathrm{g} \mathrm{L^{-1 }} h^{-1}\right)$.

When the dilution rate was reduced to $0.0050 \mathrm{~h}^{-1}$, which is equivalent to $129.3 \mu \mathrm{g} \mathrm{L}^{-1}$ $\mathrm{h}^{-1}$ nitrate addition rate, the biomass increases to $\sim 380 \mathrm{mg} \mathrm{L}^{-1}$, a constant for this dilution rate. Furthermore, the neutral lipid amount was also found to be increased from $0.0075 \mathrm{~h}^{-}$ ${ }^{1}$ dilution rate resulting in oscillatory pattern, and fluctuating between the range of $4.07 \pm$ 0.39 to $6.39 \pm 0.47 \% \mathrm{DW}$. 
(iii) Dilution rate $=0.0025 h^{-1}\left(\right.$ or $\left[\mathrm{NO}_{3}^{-}\right]$addition rate $\left.=64.7 \mu \mathrm{g} \mathrm{L}^{-1} h^{-1}\right)$

Next switch in the dilution or nitrate addition rate to the system was $0.0025 \mathrm{~h}^{-1}$ or $64.7 \mu \mathrm{g} \mathrm{L}^{-1} \mathrm{~h}^{-1}$. This resulted in further escalation in biomass growth $\left(470 \mathrm{mg} \mathrm{L}^{-1}\right)$ and accumulation of neutral lipid. It is a similar effect that was observed and discussed previously with both dilution rates $0.0075 \mathrm{~h}^{-1}$ and $0.0050 \mathrm{~h}^{-1}$. The change in neutral lipid in this dilution rate was still found to be rhythmic and changes between $6.06 \pm 0.47$ and $9.51 \pm 0.14 \mu \mathrm{g} \mathrm{L}{ }^{-1} \mathrm{~h}^{-1}$

(iv) Dilution rate $=0.0150 \mathrm{~h}^{-1}\left(\right.$ or $\left[\mathrm{NO}_{3}^{-}\right]$addition rate $\left.=388.0 \mu \mathrm{g} \mathrm{L}^{-1} \mathrm{~h}^{-1}\right)$

This dilution rate was selected to determine the effect of excess nitrate on the growth and neutral lipid production of $C$. subellipsoidea. Hence, after third limitation stage (64. 7 $\left.\mu \mathrm{g} \mathrm{L} \mathrm{L}^{-1} \mathrm{~h}^{-1}\right)$, the dilution rate was increased to mimic nitrate replete condition $\left(388.0 \mu \mathrm{g} \mathrm{L}^{-1}\right.$ $\mathrm{h}^{-1}$ ). Under this condition, within 24 hours of the shift, the cells growth and neutral lipid accumulation was found to be significantly reduced to $240 \mathrm{mg} \mathrm{L}^{-1}$ and $2.16 \pm 0.12 \mu \mathrm{g} \mathrm{L}^{-1}$ $\mathrm{h}^{-1}$, respectively. The biomass was found to be constant $-230 \mathrm{mg} \mathrm{L}^{-1}$ for the first five days, and then increased exponentially till seventh day and finally reached to a constant value of $260 \mathrm{mg} \mathrm{L}^{-1}$ for the rest three days. Further, the lipid profile follows an inverse pattern with growth, where lipid decreases from $2.16 \pm 0.12 \mu \mathrm{g} \mathrm{L}^{-1} \mathrm{~h}^{-1}$ to $1.37 \pm 0.13 \mu \mathrm{g}$ $\mathrm{L}^{-1} \mathrm{~h}^{-1}$.

The overall results suggest that growth and lipid accumulation are mutually exclusive events within a range of limited nitrate addition. This might also be the cause of the concurrent biomass and neutral lipid production in C. subellipsoidea. In literature, a similar state has been achieved by either physiological manipulation (Klok et al., 2013; 
Adams et al., 2013) or genetic engineering of lipid catabolism (Trentacoste et al., 2013) in different microalgae. Adams et al. (2013) worked on six different genus of microalgae, and found that nitrogen stress has variable effects on growth and lipid accumulation, though their studies were restricted to batch or semi-continuous mode only. Klok et al. (2013) shown a $1.5 \%$ to $12.4 \%(\mathrm{w} / \mathrm{w})$ increase in neutral lipid accumulation in Neochloris oleoabundans with maintained turbidostat. However, there is no discussion of oscillatory pattern in neutral lipid production that we have observed in our studies.

The biological significance of these rhythmic patterns in neutral lipid production is an area of research to be explored in details. Indeed, photosynthetic nature of microalgae may result in cell synchronization under continuous light. In addition, nitrogen acquisition controls the mRNA levels showing nitrogen uptake in specific phases of cell cycle (Hildebrand and Dahlin, 2000). Thus, oscillations in neutral lipid production and simultaneous biomass and lipid growth may be the result of cell synchronization and the effect of nitrogen assimilation on the growth phases of the cells. However, transcriptomics and metabolomics may be important to understand the significance of this rhythmic pattern. On the basis of our experimental results and the literature, we hypothesized that the limited nitrate acts as prey, and neutral lipid (TAG) body acts as predator, offering oscillations in lipid productivity with time.

\subsubsection{Confocal microscopy with Nile red staining}

To access the neutral lipid accumulation at different stages of dilution, confocal microscopy was used to examine the cell contents collected after every 24 hour in each dilution rate, and stained with Nile red. Nile red staining has been found an effective 
method to study the neutral lipid accumulation in the microalgae (Chen et al., 2009;

Tevatia, et al., 2012). Confocal images are presented in Figure 3, where the upper panel is the images of fluorescence, whereas the lower panel corresponds to the merged images of fluoresce and transmitted light images. All images represent the cells and/or neutral lipid body accumulated at different time points for each dilution. The TAG accumulation was found to be dependent on the nitrate rate addition. It is well known that the nitrogen stress results in neutral lipid accumulation in microalgae. When $C$. subellipsoidea is subjected to limited nitrate condition, accumulation of neutral lipid can easily be seen in Figure 3.

Further, decrease in the limited nitrate rate resulted in more lipid accumulation. However, when the nitrate addition rate was increased to the nitrate replete state, neutral lipid bodies demonstrated reduced fluorescence in accordance with the quantitative reduction of the triglyceride pool. The confocal microscopy data analysis is a qualitative measurement revealing the similar conclusion as with LC-MS/MS lipid determination.

\subsection{Modeling and fitting analysis of neutral lipid production rhythmic behavior}

\subsubsection{Model development using Lotka-Volterra model (prey-predator interaction)}

Our experimental analysis displayed that the continuous supply of limited nitrate resulted in periodic behavior of the lipid production with time. The prey-predator model has been commonly used in biological systems to define such rhythmic patterns (Demirel, 2014). We propose that the Lotka-Volterra model may be applied to our system and can be used to obtain useful parameters by fitting the experimental data. We predicted that the limited nitrate acted as prey, whereas neutral lipid bodies are 
continuously utilizing the limited nitrate. This type of relationship may have the capability to explain the oscillatory pattern that was observed in our studies.

Therefore, the interaction between limited nitrate and lipid production can be described by the following kinetics:

$$
\begin{aligned}
& \frac{d P}{d t}=k_{1} P-k_{2} P S \\
& \frac{d S}{d t}=-k_{3} S+k_{4} P S
\end{aligned}
$$

where $P$ is the amount of lipid produced and $S$ is the amount of substrate consumed. The $k_{1}$ and $k_{3}$ are the differences in lipid production and nitrate consumption, respectively. The terms $k_{2}$ and $k_{4}$ represents the interaction between nitrate utilization and lipid body production. Stationary state solutions of Eq (1) and (2) were obtained by equating $d P / d t$ and $d S / d t$ to 0 , which resulted in two solutions. One of the solution is $P=S=0$, and the second solution is:

$$
P_{S}=\frac{k_{3}}{k_{4}}, \text { and } S_{S}=\frac{k_{1}}{k_{2}}
$$

In order to study whether these states are stable or not, a small perturbations $\delta P$ and $\delta S$ were applied to stationary state solutions, so that $P=P_{S}+\delta P$ and $S=S_{S}+\delta S$, where $P_{\mathrm{s}}$ and $S_{\mathrm{s}}$ are the steady state concentration of product and substrate, respectively. We can introduce these expressions in Eq. (1) and (2), and neglect very small changes due to the product of $\delta P$ and $\delta S$. The perturbed kinetics can be expressed as:

$$
\frac{d(\delta P)}{d t}=k_{1} \delta P-k_{2}\left(P_{S} \delta S+S_{S} \delta P\right)
$$




$$
\frac{d(\delta S)}{d t}=-k_{3} \delta S+k_{4}\left(P_{S} \delta S+S_{S} \delta P\right)
$$

Using $k_{1}$ and $k_{3}$ from Eq. (3), Eqs. (4) and (5) can be rewritten as:

$$
\begin{aligned}
& \frac{d(\delta P)}{d t}=-k_{2} P_{S} \delta S \\
& \frac{d(\delta S)}{d t}=k_{4} S_{S} \delta P
\end{aligned}
$$

Differentiating Eq. (6) once, we obtain:

$$
\frac{d^{2}(\delta P)}{d t^{2}}=-k_{2} P_{S} \frac{d(\delta S)}{d t}
$$

After equating the value of $d(\delta S) / d t$ from Eq. (7), Eq. (8) reduces to:

$$
\frac{d^{2}(\delta P)}{d t^{2}}=-k_{2} k_{4} P_{S} S_{S} \delta P=-k_{1} k_{3} \delta P
$$

Equation (9) has the form of motion of a harmonic oscillator, and the solution yields a harmonic oscillation:

$$
\delta P=\delta P_{0} \cos (2 \pi f t)
$$

and

$$
\frac{d(\delta P)}{d t}=-\delta P_{0} 2 \pi f \sin (2 \pi f t)
$$

where $f$ is the frequency and given by $f=\left(\frac{1}{2 \pi}\right)\left(k_{2} k_{4} P_{S} S_{S}\right)^{1 / 2}=\left(\frac{1}{2 \pi}\right)\left(k_{1} k_{3}\right)^{1 / 2}$. Therefore, nitrate limitation conditions resulted in the unstable microalgae lipid production, which behaves like an oscillatory system with frequency $f$. This frequency can be used to determine the relationship between the lipid production and nitrate consumption rates. The Eq. (11) can be reorganize with initial condition $P(t=0)=P_{0}$ : 


$$
\frac{d P(t)}{d t}=P_{0} f_{c}-\left(P_{0}-P_{S}\right) 2 \pi f \sin (2 \pi f t)
$$

where $f_{c}$ is frequency constant used due to expansion of $\delta P$.

The non-dimensionalized Eq. (12) can be represented as:

$$
\frac{d \theta_{P}}{d \tau}=1-\left[\left(1-\theta_{P S}\right)(2 \pi \eta \sin (2 \pi \eta \tau))\right]
$$

where $\theta_{P}=\frac{P}{P_{0}}, \theta_{P S}=\frac{P_{S}}{P_{0}}, \tau=f_{c} t$, and $\eta=\frac{f}{f_{c}}$.

Therefore, the lipid productivity oscillates with time and varies with respect to dilution rate. Fitting of Eq. (13) could help in obtaining parameters having a meaning in terms of interaction between limited nitrate consumption and lipid body production. The Eq. (13) was used to fit the non-dimensional data for lipid productivity with time.

\subsubsection{Model fitting and sensitivity analysis}

The model Eq. (13) relates the instability in the lipid productivity results in the oscillations. Eq. (13) was fitted with lipid productivity with time using Mathematica 9.0.1.0 (Wolfram Research Inc., USA). All the values of lipid productivity and time were changed according to non-dimensionalized parameters in Eq. (13) to ease in the model fitting, and allowed us in evaluating the values of constants, significant for scale-up. Figure 4 shows the fitting of experimental data with model Eq. (13). The parameters obtained after fitting are listed in Table 1. Further, sensitivity analysis was done in order to observe that the experimental data are sufficient enough for estimating the parameters using the modeling equation. For this purpose, ANOVA (Analysis of Variance) sensitivity analysis was performed using Mathematica. 
The frequency of oscillations was found to be decreasing with decreasing nitrate addition rates. This signifies that the time period of each oscillation was increasing with decrease in nitrate rates, i.e. the decrease in nitrate addition rate from $194.0 \mu \mathrm{g} \mathrm{L}^{-1} \mathrm{~h}^{-1}$ to 129.3 $\mu \mathrm{g} \mathrm{L}^{-1} \mathrm{~h}^{-1}$, and subsequently to $64.7 \mu \mathrm{g} \mathrm{L}^{-1} \mathrm{~h}^{-1}$ results in the increase in oscillation time period from 6.45 days to 9.85 days, and then to 11.89 days, respectively. The above observations indicates that (i) the neutral lipid accumulation is highest for the lowest amount of continuous limited nitrate addition, and (ii) the time taken to achieve maximum lipid accumulation is low when nitrate addition rate is high. Therefore, it is an important decisive point to select either less time to achieve high lipid production or maximum attainable neutral lipid productivity, where the advisable will be to have the situation that results in the maximum neutral lipids yields. Though, our system is a continuous process, low dilutions might be a problem for scale up. However, our model may help in choosing the time points (by observing the time periods of these oscillations) where the cells with maximum lipid amounts can be harvested from system the most.

\subsection{Effect of nitrate limitation on maximum growth and neutral lipid productivity}

\subsubsection{Biomass growth in a turbidostat photobioreactor}

\subsubsection{Biomass growth in a turbidostat photobioreactor}

The modeling equation for microalgae biomass production in a continuous photobioreactor can be modeled by logistic approach and represented as:

$$
\frac{d X}{d t}=-D X+\mu_{m}\left(1-\frac{X}{X_{m}}\right) X
$$


where $X$ is cells concentration (biomass), $D$ is the dilution rate, $\mu_{m}$ is maximum specific growth rate, and $X_{m}$ is maximum biomass achieved. Under steady state condition or turbidostat condition $(d X / d t=0)$, the biomass is given by:

$$
X=-\left(\frac{X_{m}}{\mu_{m}}\right) D+X_{m}
$$

The numerical values for the constants $\left(X_{m}\right.$ and $\left.\mu_{m}\right)$ in Eq. (15) can be obtained by plotting the change in biomass production with varied dilution rates. Intercept of this equation will be the maximum biomass, and slope can be used to estimate maximum specific growth under given nitrate-limited conditions.

\subsubsection{Maximum biomass and neutral lipid productivity}

The maximum amounts of biomass and lipid accumulation were calculated by plotting the respective values with dilution rate (Fig. 5a). The intercept and slope of Eq. (15) were used to calculate the maximum biomass concentration $\left(X_{m}=509.3 \mathrm{mg} \mathrm{L}^{-1}\right)$ and maximum specific growth rate $\left(\mu_{m}=0.022 \mathrm{~h}^{-1}\right)$ for $C$. subellipsoidea under these nitrate limitation conditions. A similar approach was applied for lipid accumulation, where the intercept obtained after linear fitting was the maximum amount of lipid accumulation (9.96 \% DW - Dry Weight). Recently, Allen et al. (2014) observed that batch production of C. subellipsoidea accumulates maximum TAG amount of $6.62 \%$ and $17.6 \% \mathrm{DW}$ on $3^{\text {rd }}$ and $7^{\text {th }}$ day of nitrogen starvation, respectively. Further, continuous turbidostat study done by Klok et al. (2013) shows $12.4 \%$ DW neutral lipid accumulation in Neochloris oleoabundans. 
Likewise, the values of maximum biomass productivity $\left(P_{X m}=120.1 \mathrm{mg} \mathrm{L}^{-1} \mathrm{~h}^{-1}\right)$ and neutral lipid productivity $\left(P_{P m}=94.97 \mu \mathrm{g} \mathrm{L}^{-1} \mathrm{~h}^{-1}\right)$ were calculated by plotting and fitting the respective values with respect to nitrate addition rates (Fig. 5b). These physiological parameters are useful during the scale up.

\subsection{Model limitations and consequences}

The prey and predator model was applied to develop the model used in the present study, which was assumed on the basis of experimental data (oscillations), and literature (Smouse, 1981; Demirel, 2014). Though, the model simulated lipid rhythmic production, only the physiological significance of the pattern was obtained. In order to have better understanding, biological significance of the oscillatory patterns must be acquired by high throughput transcriptomics, proteomics, and/or metabolomics. Further, this model applicability is defined for a range of limitation, hence under replete or deplete/starved conditions, different models and assumption must be used. Therefore, a universal model to define any system is still in demand.

The present work is a continuous process offering a major advantage of higher daily biomass and lipid productivity over batch or semi-continuous processes. As most of the scaled microalgal growth systems are open and batch (raceway ponds) (Chisti and Yan, 2011; Brennan and Owende, 2010), the application of this continuous system requires a systematic arrangements. The model predictions helped in concluding that each nitrate addition rate results in distinctive neutral lipid oscillations. Lower amount of limited nitrate condition results in higher biomass and lipid content, however oscillation studies predicted that the time required to achieve this state is greater than the time period of 
oscillations of the highest amount of limited nitrate addition. Henceforth, choosing a right strategy may be helpful in defining a better production system. Moreover, this is a fist step in achieving superior amount of total lipid productivity, but there are several other factors like harvesting, and conversion of microalgal oil to biofuel to be used as deciding factors for production of low cost microalgal based biofuel.

The forte of this study might not be in commercialization, but may be in understanding its biological significance. The experimental set up allowed the cells mostly packed with neutral lipids to divide. Further, analysis of these oscillations might be an extensive and rewarding area of research. As per our modeling approach and experimental results, these oscillations may be a result of instability in neutral lipid production. This creates lipid production as a mutually exclusive event, separating from the biomass production. Furthermore, the results generated by these experiments are very suitable for transcriptomics, proteomics, and metabolomics studies for improving understanding of the regulation of TAG accumulation in the microalgae.

\section{Conclusions}

Generally, nitrogen stress results in inconsistent biomass and neutral lipid production in microalgae; however, using the present experimental approach, the simultaneous production of biomass and neutral lipid was achieved. The continuous addition of limited nitrate was found to generate oscillations in lipid productivity with time. Though we were able to capture physiological meaning of these oscillations, further analysis will be needed to understand the respective biological significance. The most promising microalgae might be a strain that will combine the characteristics to accumulate high 
neutral lipids in growing microalgae.

Acknowledgements. This research was supported by a grant from the Department of Energy National Science Foundation (EPSCOR) (grant number: DE-FG36-08GO88055), and Office of Research and Economic Development, University of Nebraska, Lincoln, $\mathrm{NE})$.

\section{References}

1. Adams, C., Godfrey, V., Wahlen, B., Seefeldt, L., Bugbee, B., 2013. Understanding precision nitrogen stress to optimize the growth and lipid content tradeoff in oleaginous green microalgae, Bioresour. Technol. 131, 188-194.

2. Allen, J.W., DiRusso, C.C., Black, P.N., 2014. Triglyceride quantification by catalytic saturation and LC-MS/MS reveals an evolutionary divergence in regioisometry among green microalgae, Algal Research. 5, 23-31.

3. Bligh, E.G., Dyer, W.J., 1959. A rapid method of total lipid extraction and purification, Can. J. Biochem. Physiol. 37, 911-917.

4. Bold, H.C., 1949. The morphology of Chlamydomonas chlamydogama sp. nov. Bull. Torrey Bot. Club. 76, 101-108.

5. Brennan, L., Owende, P., 2010. Biofuels from microalgae-A review of technologies for production, processing, and extractions of biofuels and co-products, Renew. Sust. Energ. Rev. 14, 557-577.

6. Breuer, G., Lamers, P.P., Martens, D.E., Draaisma, R.B., Wijffels, R.H., 2012. The impact of nitrogen starvation on the dynamics of triacylglycerol accumulation in nine microalgae strains, Bioresour. Technol. 124, 217-226.

7. Chen, W., Zhang, C., Song, L., Sommerfeld, M., Hu, Q., 2009. A high throughput Nile red method for quantitative measurement of neutral lipids in microalgae. J. Microbiol. Methods. 77, 41-47.

8. Chisti, Y., Yan, J., 2011. Energy from algae: Current status and future trends, Appl. Energ. 88, 3277-3279.

9. Das, P., Aziz, S.S., Obbard, J.P., 2011. Two phase microalgae growth in the open system for enhanced lipid productivity, Renew. Energ. 36, 2524-2528. 
10. Demirel, Y., 2014. Non-equilibrium Thermodynamics: Transport and Rate Processes in Physical, Chemical and Biological Systems, 3rd ed. Elsevier, Amsterdam.

11. Goldbeter, A., 2002. Computational approaches to cellular rhythms, Nature. 420, 238-245.

12. Gouveia, L., Oliveira, A.C., 2009. Microalgae as a raw material for biofuels production, J. Ind. Microbiol. Biotechnol. 36, 269-274.

13. Hildebrand, M., Dahlin, K., 2000. Nitrate transporter genes from the diatom Cylindrotheca fusiformis (bacillariophyceae): mRNA levels controlled by nitrogen source and by the cell cycle, J. Phycol. 36, 702-713.

14. Hoffmann, M., Marxen, K., Schulz, R., Vanselow, K.H., 2010. TFA and EPA productivities of Nannochloropsis salina influenced by temperature and nitrate stimuli in turbidostatic controlled experiments, Mar. Drugs. 8, 2526-2545.

15. Klok, A.J., Martens, D.E., Wijffels, R.H., Lamers, P.P., 2013. Simultaneous growth and neutral lipid accumulation in microalgae, Bioresour. Technol. 134C, 233-243.

16. Lacour, T., Sciandra, A., Talec, A., Mayzaud, P., Bernard, O., 2012. Neutral lipid and carbohydrate productivities as a response to nitorgen status in Isochrysis sp. (T- iso; haptophyceae): starvation versus limitation. J. Phycol. 48, 647-656.

17. Prathima Devi, M., Venkata Subhash, G., Venkata Mohan, S., 2012. Heterotrophic cultivation of mixed microalgae for lipid accumulation and wastewater treatment during sequential growth and starvation phases: Effect of nutrient supplementation, Renew. Energ. 43, 276-283.

18. Richardson, B., Orcutt, D.M., Schwertner, H.A., Martinez, C.L., Wickline, H.E., 1969. Effects of nitrogen limitation on the growth and composition of unicellular algae in continuous culture, Appl. Microbiol. 18, 245-250.

19. Smouse, P.E., 1981. Mathematical models for continuous culture growth dynamics of mixed populations subsisting on a heterogeneous resource base. II. Predation and trophic structure, Theor. Popul. Biol. 20, 127-149.

20. Tevatia, R., Demirel, Y., Blum, P., 2012. Kinetic modeling of photoautotropic growth and neutral lipid accumulation in terms of ammonium concentration in Chlamydomonas reinhardtii, Bioresour. Technol. 119, 419-424.

21. Trentacoste, E.M., Shrestha, R.P., Smith, S.R., Gle, C., Hartmann, A.C., Hildebrand, M., Gerwick, W.H., 2013. Metabolic engineering of lipid catabolism increases microalgal lipid accumulation without compromising growth, Proc. Natl. Acad. Sci. U. S. A. 110, 19748-19753. 
22. Yeh, K., Chang, J., 2011. Nitrogen starvation strategies and photobioreactor design for enhancing lipid content and lipid production of a newly isolated microalga Chlorella vulgaris ESP-31: Implications for biofuels, Biotechnol. J. 6, 1358-1366. 
Table 1. Various parameters used and estimated after fitting the model.

\begin{tabular}{lllll}
\hline Parameters & Units & \multicolumn{2}{c}{ Continuous Limited Nitrate Addition } \\
\cline { 3 - 5 } & & Dilution 1 & Dilution 2 & Dilution 3 \\
\hline${ }^{\mathrm{a}}$ Dilution Rate, $D$ & $\mathrm{~h}^{-1}$ & 0.0075 & 0.005 & 0.0025 \\
${ }^{\mathrm{a}}$ Nitrate addition rate & $\mu \mathrm{g} \mathrm{L}^{-1} \mathrm{~h}^{-1}$ & 194.0 & 129.3 & 64.7 \\
${ }^{\mathrm{a}}$ Frequency constant, $f_{c}$ & $\mathrm{Day}^{-1}$ & 0.1 & 0.1 & 0.1 \\
${ }^{\mathrm{a}}$ Initial Lipid Production, $P_{0} \mathrm{a}$ & $\mu \mathrm{g} \mathrm{L}^{-1} \mathrm{~h}^{-1}$ & $39.65 \pm 4.44$ & $47.18 \pm 2.18$ & $79.14 \pm 5.01$ \\
${ }^{\mathrm{b}}$ Frequency of oscillation, $f$ & $\mathrm{Day}^{-1}$ & $0.155 \pm 0.003$ & $0.102 \pm 0.002$ & $0.084 \pm 0.003$ \\
${ }^{\mathrm{b}}$ Time Period of oscillation & $\mathrm{Days}^{\mathrm{b}}$ & 6.45 & 9.85 & 11.89 \\
${ }^{\mathrm{b}}$ Steady state Lipid production, $P_{S}$ & $\mu_{\mathrm{g} \mathrm{L}} \mathrm{L}^{-1} \mathrm{~h}^{-1}$ & $39.17 \pm 4.09$ & $45.52 \pm 1.40$ & $76.45 \pm 3.12$ \\
${ }^{\mathrm{b}} R^{2}$ & - & 0.999 & 0.998 & 0.999
\end{tabular}

${ }^{\mathrm{a}}$ - Experimentally calculated data

${ }^{b}$ - Model fit parameters calculated using Mathematica 
Figure 1. Schematic representation of continuous stirred photobioreactor experimental setup. 1) Freezer $\left(4^{\circ} \mathrm{C}\right)$ for continuous storage of outlet samples; 2$)$ input vessel containing BBM as inflow medium; 3) computer with bioexpert software for online data collection; 4) peristaltic pump; 5) ez-controller; 6) biomass monitor; 7) $\mathrm{pH}$ probe; 8) DO probe; 9) continuous light source; 10) stirrer assembly using Rushton turbine; 11) exit gases; 12) condenser; 13) 3L Applikon photobioreactor with $2 \mathrm{~L}$ working volume, controlled $\mathrm{pH}$, agitation, and aeration; 14 ) chiller.

Figure 2. Plot summarizing the overall changes in dry weight (mg/L) and TAG accumulation (\% Dry Weight) with time (days) during continuous photobioreactor limitation experiments implemented with four different nitrate input rates (standard error shown for three replicates).

Figure 3. Confocal microscopy for the detection of TAG accumulation in Coccomyxa sp. C169 with different nitrate addition rates. The upper panel represents the fluorescent images (red dots signifies nile red stained lipid bodies) and lower panel shows the merged transmitted light and fluorescent images accounting for the cell population with lipid bodies.

Figure 4. Fitting of experimental data (three replicates) for Coccomyxa sp. C169 grown photoautotropically at different nitrate limitation rates a) $194.0 \mu \mathrm{g} \mathrm{L}^{-1} \mathrm{~h}^{-1}$ ( Experimental data, — Model Fitting), b) $129.3 \mu \mathrm{g} \mathrm{L}^{-1} \mathrm{~h}^{-1}$ ( Experimental data, . . Model Fitting), and c) 64.7 $\mu \mathrm{g} \mathrm{L}^{-1} \mathrm{~h}^{-1}$ ( $\Delta$ Experimental data, - - - Model Fitting) with respective modeled equation representing the oscillations. Experimental data points were normalized to dimensionless neutral lipid productivity and time values, which facilitate better comparisons and avoid any round off experimental errors.

Figure 5. Linear fitting of a) dry weight (mg/L) and neutral lipid (\% dry weight) with dilution rates, and b) biomass and neutral lipid productivity with nitrate addition rates. 


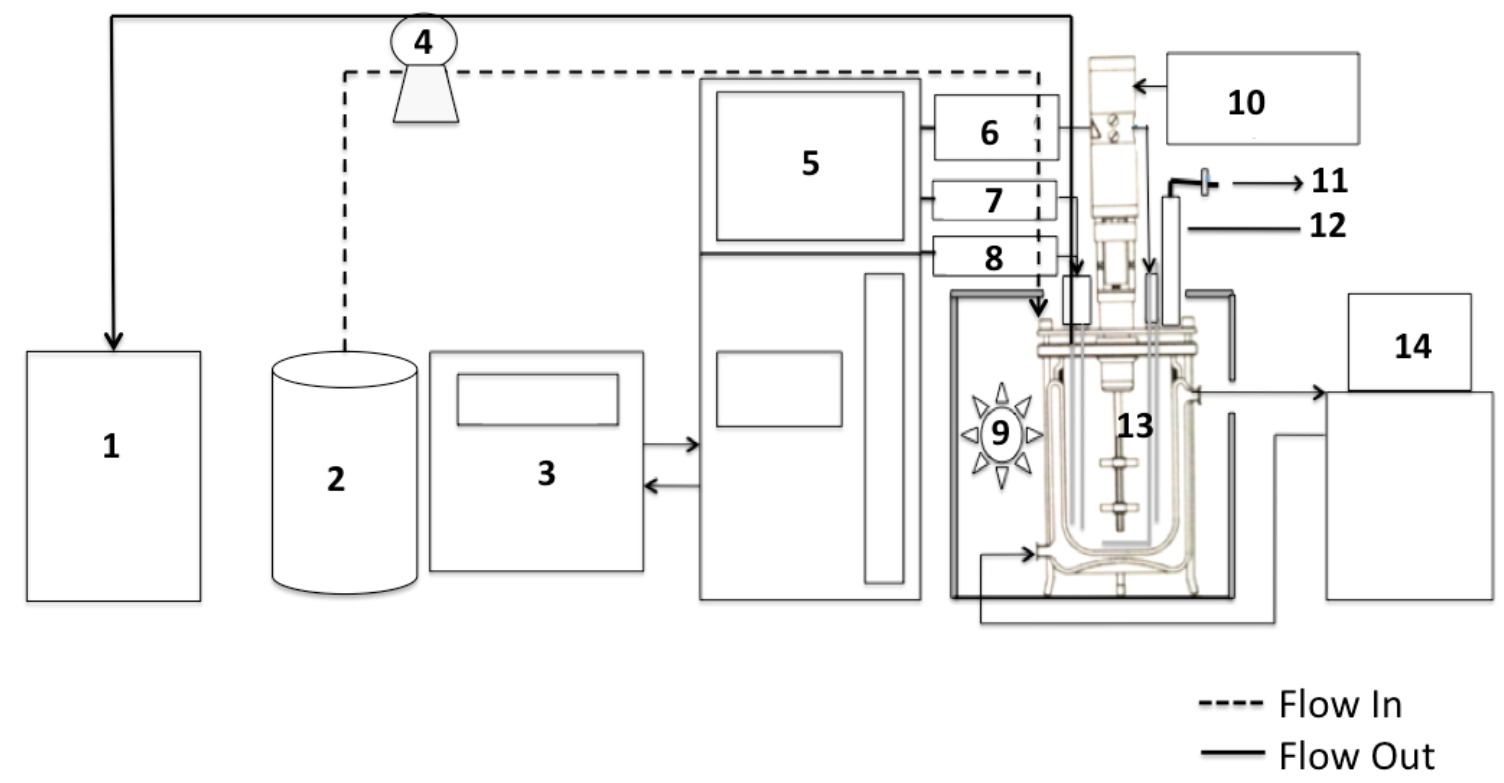

Figure 1. 


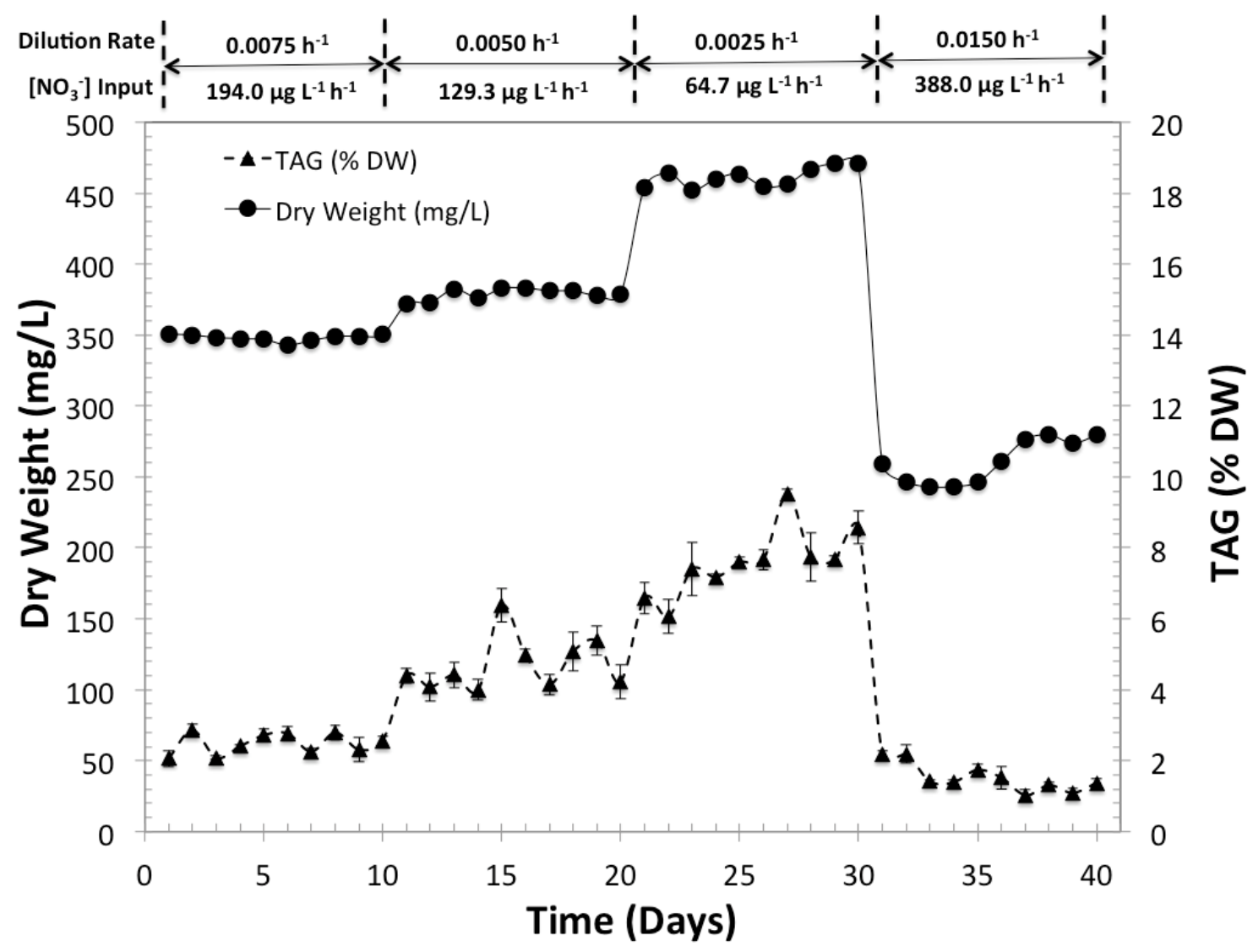

Figure 2. 


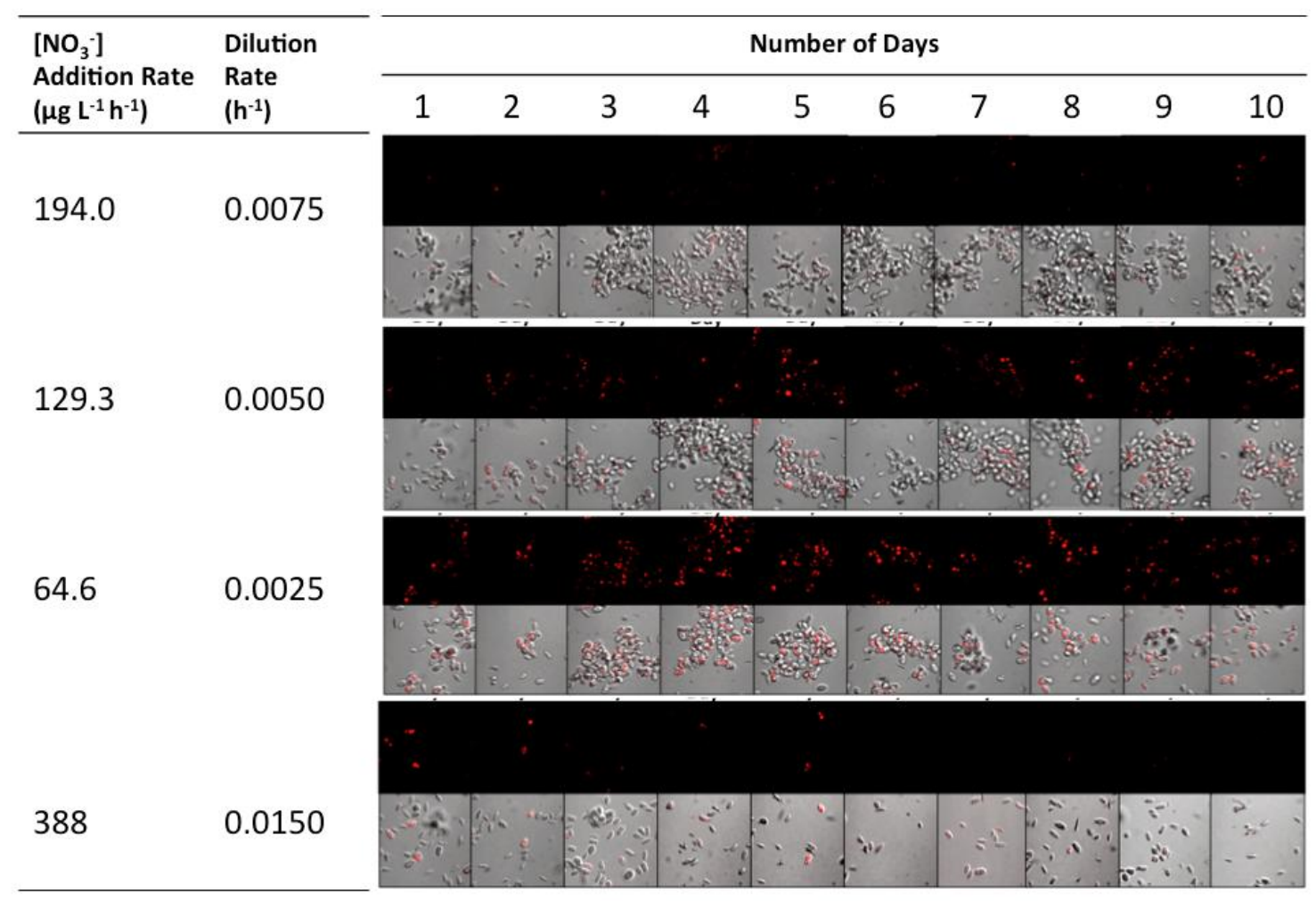

Figure 3. 


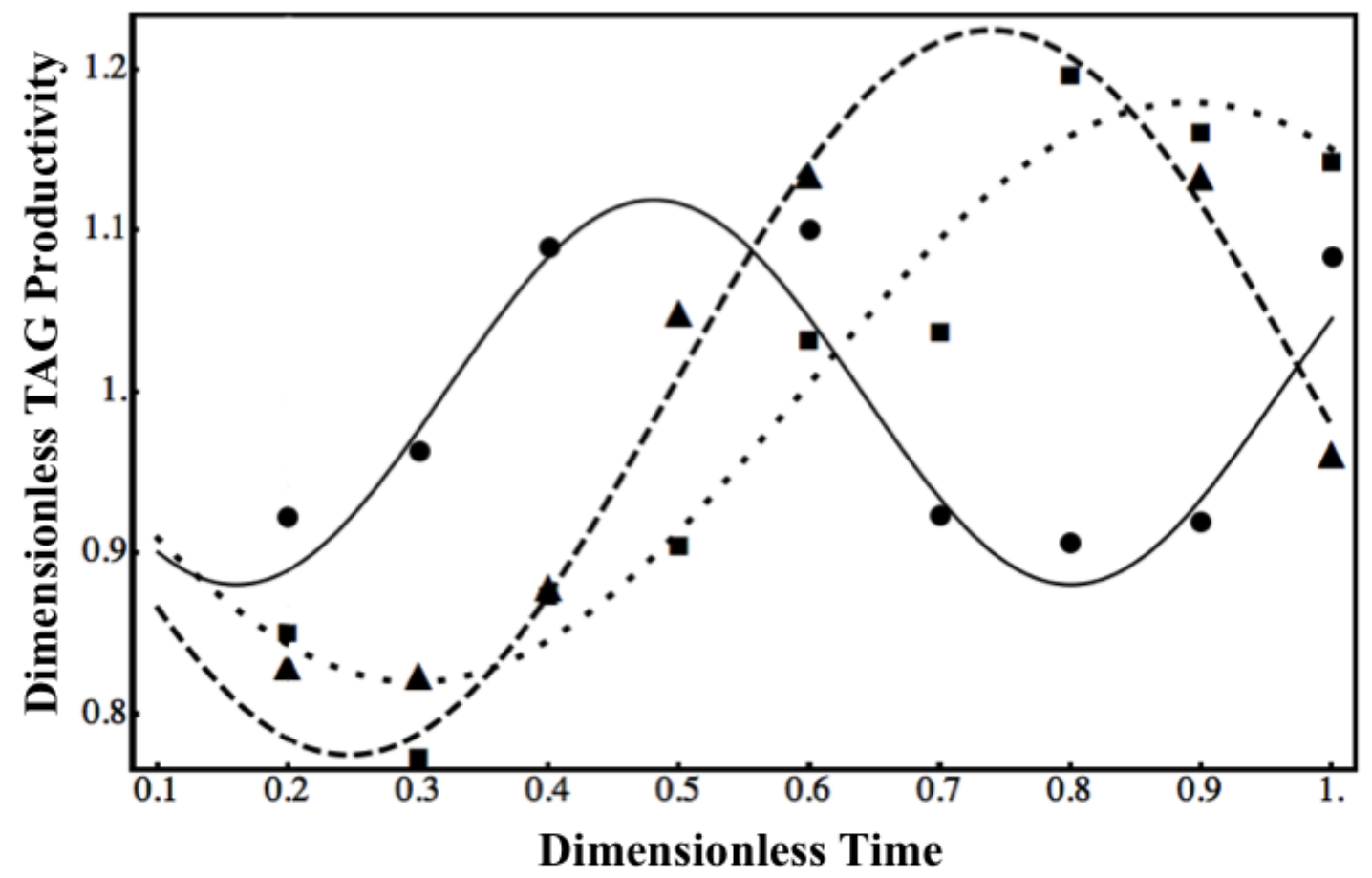

Figure 4. 
a)



b)

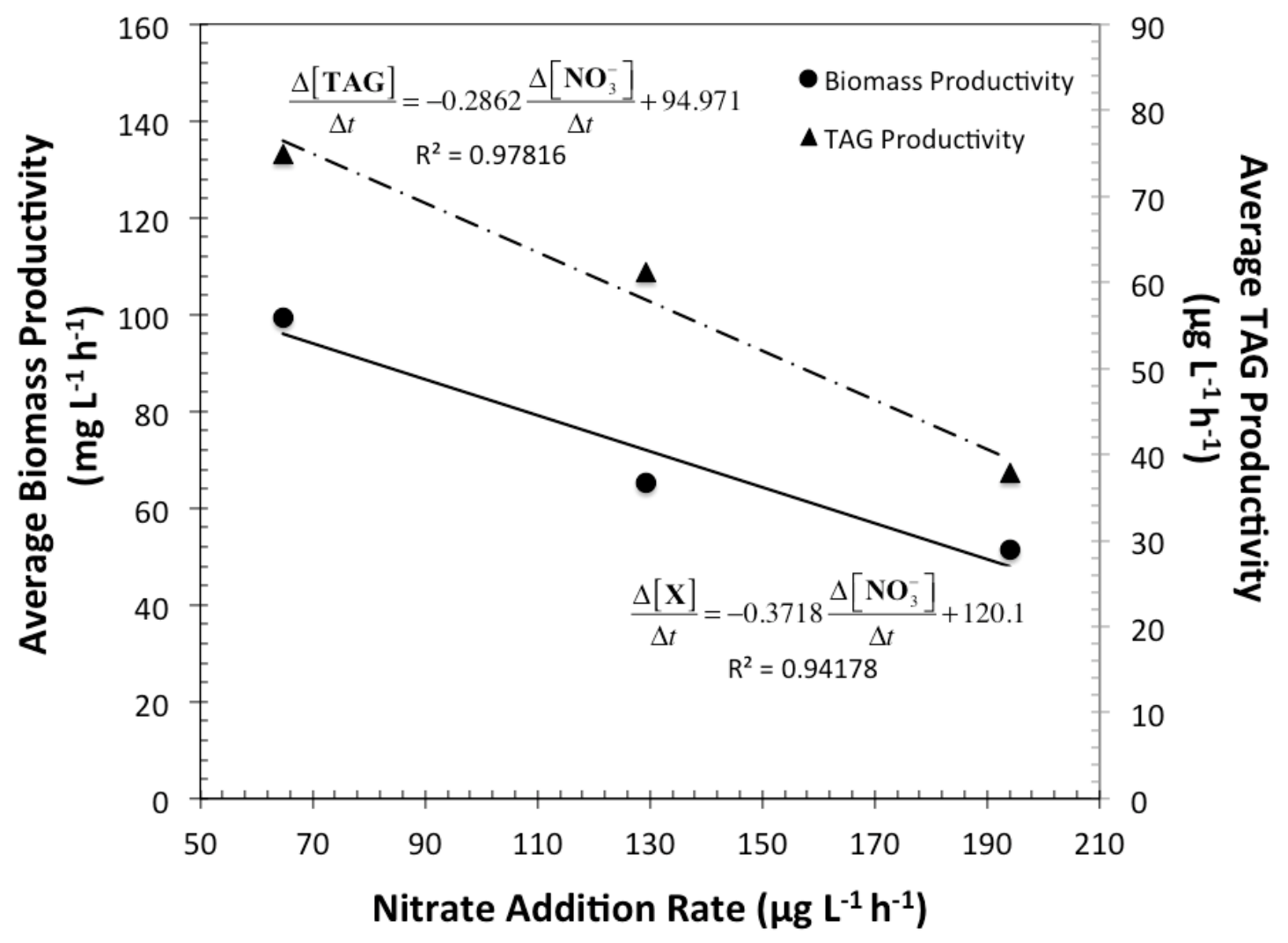

Figure 5. 
Graphical Abstract

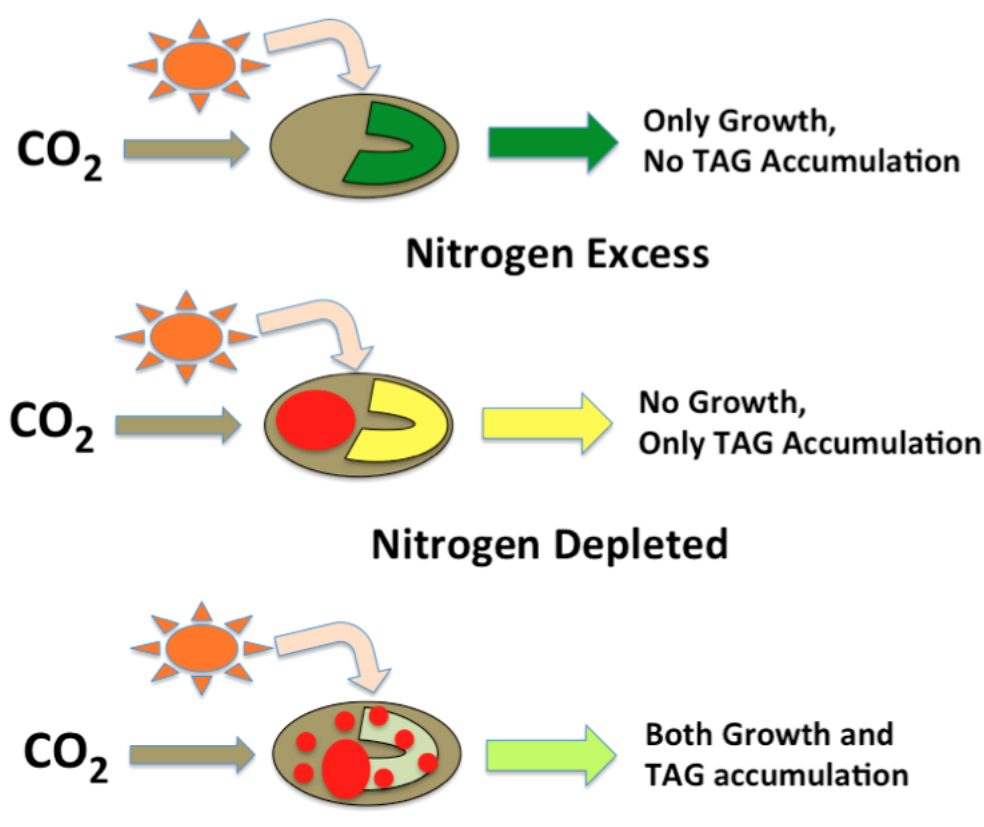

Nitrogen Limited

\author{
Continuous \\ Neutral Lipid \\ Production
} Neutral Lipid Production

Batch Neutral Lipid Production 